\title{
OPEN Compromised balance control in older people with bilateral medial knee osteoarthritis during level walking
}

\author{
Pei-An Lee ${ }^{1}$, Kuan-Hsien Wu ${ }^{1}$, Hsuan-Yu Lu ${ }^{1}$, Kai-Wen Su ${ }^{1}$, Ting-Ming Wang ${ }^{1,3}$, \\ Hwa-Chang Liu ${ }^{2 \bowtie}$ \& Tung-Wu Lu ${ }^{1,3 \bowtie}$
}

\begin{abstract}
About half of the elderly population has knee osteoarthritis (OA), showing altered gait patterns with increased fall risk. The current study aimed to identify the effects of severe bilateral medial knee osteoarthritis on gait balance control, in terms of the inclination angle (IA) of the center of pressure to center of mass vector, and the rate of change of IA (RCIA). Fifteen older adults with severe bilateral medial knee $O A$ and 15 healthy controls walked at their preferred walking speed while the kinematic and forceplate data were measured to calculate IA, RCIA and temporal-spatial parameters. The OA group showed compromised gait balance control, due to a decreased average and range of sagittal RCIA over double-limb support (DLS, $p<0.002$ ) and single-limb support ( $S L S, p<0.002$ ), as well as an increased sagittal IA (DLS, $p<0.005$ ). Significantly decreased frontal RCIA averages during DLS, heelstrike and toe-off, and decreased RCIA ranges during SLS and swing $(p<0.027)$ were also observed. Reducing RCIA during DLS appeared to help reduce the loading rate and pain at the knees, and reduced RCIA at the subsequent SLS. The results indicated an increased risk of loss of balance in the OA group, and may warrant regular monitoring for reduced RCIA during gait to determine fall risk.
\end{abstract}

Musculoskeletal deteriorations are closely related to balance and gait problems, a major contributor to accidental falls in the older population ${ }^{1-3}$. A musculoskeletal disorder most prevalent in the elderly is knee osteoarthritis (OA), a degenerative disease in which the joint cartilage wears away progressively $y^{4,5}$. About $50 \%$ of the elderly population above 60 years old have knee $\mathrm{OA}^{4,6,7}$, resulting in restricted physical activities with pain and symptoms such as joint swelling and stiffness, muscle weakness, and limited range of motion ${ }^{8}$. These symptoms and the accompanied alterations in joint biomechanics have a direct impact on the gait patterns and balance control ${ }^{9-12}$. Therefore, knowledge of the balance control during gait in people with knee OA may provide useful information for the management of fall risks in this patient population.

Previous studies have reported decreased standing postural stability in older people with unilateral and bilateral knee $\mathrm{OA}^{12-15}$. However, clinical observations indicated that falls most frequently occur during activities involving larger displacement of the body's center of mass (COM), such as walking ${ }^{16}$. Therefore, quantifying standing postural stability alone is not sufficient to describe dynamic balance, and monitoring of the motion of the COM is essential.

Quantifying the motion of the body's COM relative to the center of pressure (COP) has been used to evaluate the body's balance control during locomotion ${ }^{17,18}$. A common measure is the COM-COP separation defined by the horizontal distance between the COP and the vertical projection of the COM on the ground. In contrast to static balance during which the downward vertical projection of the COM has to be kept close to the COP, during walking the projected COM can be further away from the COP without loss of balance as long as the COM is controlled at an appropriate velocity relative to the $\mathrm{COP}^{19}$. It follows that the $\mathrm{COM}-\mathrm{COP}$ separation alone cannot indicate the actual control of the COM relative to the COP, as it does not consider the velocity of the COM relative to the COP, nor the influence of the body height or leg length. To overcome the limitations of COM-COP separations, another approach that uses the COM-COP inclination angle (IA) formed by the COM-COP vector and the vertical, and the rate of change of IA (RCIA) has been proposed. The IA combines both the information

${ }^{1}$ Department of Biomedical Engineering, National Taiwan University, Taipei, Taiwan, ROC. ${ }^{2}$ Department of Orthopaedic Surgery, Taiwan Adventist Hospital National Taiwan University Hospital, Taipei, Taiwan, ROC. ${ }^{3}$ Department of Orthopaedic Surgery, School of Medicine, National Taiwan University, Taipei, Taiwan, ROC. ${ }^{\boxplus}$ email: hcliu8@gmail.com; twlu@ntu.edu.tw 


\begin{tabular}{|l|l|l|l|}
\hline & OA & Control & p value \\
\hline Female/male & $12 / 3$ & $12 / 3$ & - \\
\hline Age (years) & $66.8(7.2)$ & $66.5(7.4)$ & 0.903 \\
\hline Body height $(\mathrm{cm})$ & $155.7(6.6)$ & $156.1(5.7)$ & 0.888 \\
\hline Body mass $(\mathrm{kg})$ & $67.3(14.7)$ & $61.9(10.7)$ & 0.279 \\
\hline BMI & $27.5(4.4)$ & $25.4(4.2)$ & 0.189 \\
\hline Tibiofemoral angle (degrees) & $-3.4(2.9)$ & - & - \\
\hline
\end{tabular}

Table 1. Means (standard deviations) of the demographic characteristic of subjects in the knee osteoarthritis group $(\mathrm{OA}, \mathrm{n}=15)$ and healthy controls (Control, $\mathrm{n}=15)$. ${ }^{\star} \mathrm{OA}$ significantly different from Control.

of COM-COP separation and the height of the COM, and the RCIA integrates the information of the velocity of the COM relative to the COP. The IA and RCIA together provide a more complete description of the COM-COP control, and better enable comparisons between people of different statures ${ }^{17,20,21}$. Generally, interpretation of the IA and RCIA data of a patient group should be made with reference to those of healthy controls in order to identify any particular balance control strategies adopted. For example, an increased IA may suggest a poor balance control, but if one is able to generate an appropriate RCIA either by changing the velocity of the COM or that of the COP or both, the combined changes are considered a successful balance control strategy.

The study of balance control strategies during dynamic activities in terms of IA and RCIA has been reported in various populations ${ }^{18,20,22}$. However, previous studies on patients with knee OA have been limited, and have focused mainly on the COM and IA without considering the velocities of the COM relative to the COP ${ }^{23,24}$. Mandeville et al. ${ }^{23}$ studied the balance control in patients before and after unilateral total knee replacement, examining the maximum COM-COP inclination angles without considering data at gait events and during phases, nor the velocities of the COM relative to the COP. These studies have limited the correct interpretation of the COM-COP data of the knee OA patient group. To the best knowledge of the authors, no study has investigated the effects of knee OA on the balance control in terms of IA and RCIA of the COM motions relative to the COP during level walking.

The purpose of the current study was to identify the effects of bilateral medial knee OA on whole body balance control in older people during level walking, in terms of IA and RCIA. It was hypothesized that older people with severe medial OA would walk with altered, more conservative COM-COP control with increased IA but decreased RCIA when compared to healthy controls.

\section{Methods}

All experiments of the current study were conducted under the approval of Taiwan Adventist Hospital Institutional Review Board (IRB No. 106-E-15). All the experiments and procedures conformed to the Ethical Principles for Medical Research Involving Human Subjects (World Medical Association Declaration of Helsinki). Fifteen older patients with bilateral medial knee OA (OA group), and 15 healthy controls (Control group) matching with the OA group for sex, age and BMI participated in the current study with written informed consent (Table 1). All the patients were determined radiographically to have severe bilateral medial knee OA (KL grade 4) by a senior consultant orthopaedic surgeon with more than 30 years of experience (HCL) using Kellgren and Lawrence criteria $^{25}$ (Table 1). They were also assessed via the Western Ontario and McMaster Universities Osteoarthritis Index (WOMAC) questionnaire. Participants were excluded from the study if they had received an intra-articular corticosteroid injection in the previous two months, or if they had other neuromusculoskeletal diseases or neurological pathology that might affect gait. An a priori power analysis for a two-group independent sample $t$ test for the comparison of IA and RCIA between older adults with knee OA and healthy controls based on pilot results using GPOWER ${ }^{26}$ determined that a projected sample size of five subjects for each group would be needed with a power of 0.8 and a large effect size (Cohen's $d=0.8$ ) at a significance level of 0.05 . Thus, 15 subjects for each group were more than adequate for the main objectives of the current study.

In a gait laboratory, each subject walked at a self-selected, comfortable pace on a $10-\mathrm{m}$ walkway. The motions of their body segments were tracked by 39 infrared retro-reflective markers placed on specific anatomical landmarks, namely ASISs, PSISs, greater trochanters, mid-thighs, medial and lateral epicondyles, heads of fibulae, tibial tuberosities, medial and lateral malleoli, navicular tuberosities, fifth metatarsal bases, big toes and heels, and mandibular condylar processes, acromion processes, C7, medial and lateral humeral epicondyles, and ulnar styloids ${ }^{21,27,28}$. Three-dimensional (3D) trajectories of the markers were measured using an 8-camera motion analysis system (Vicon MX T-40, UK) at $120 \mathrm{~Hz}$ while the ground reaction forces (GRF) were measured simultaneously at $1080 \mathrm{~Hz}$ using three forceplates $(50.8 \mathrm{~cm} \times 46.2 \mathrm{~cm}$, OR-6-7-1000, AMTI, USA). The motion analysis system has been shown to have a high precision and accuracy with a mean absolute error of $0.15 \mathrm{~mm}$ and a variability lower than $0.025 \mathrm{~mm}^{29}$, which was considered appropriate for the purpose of the current study. The subjects were allowed to walk on the walkway several times before data collection. Data of a total of six trials, each containing a complete gait cycle for each lower limb, were obtained for each subject.

From the measured marker and GRF data, the mass and position of the COM for each of the body segments were obtained using an optimization-based $\operatorname{method}^{28}$, and the body's COM was calculated as the mass-weighted sum of the COMs of all body segments using a 13-body-segment model. This approach has been shown to have reduced errors in the calculated COM motion when compared to traditional prediction methods ${ }^{28}$. The COP 
(a)
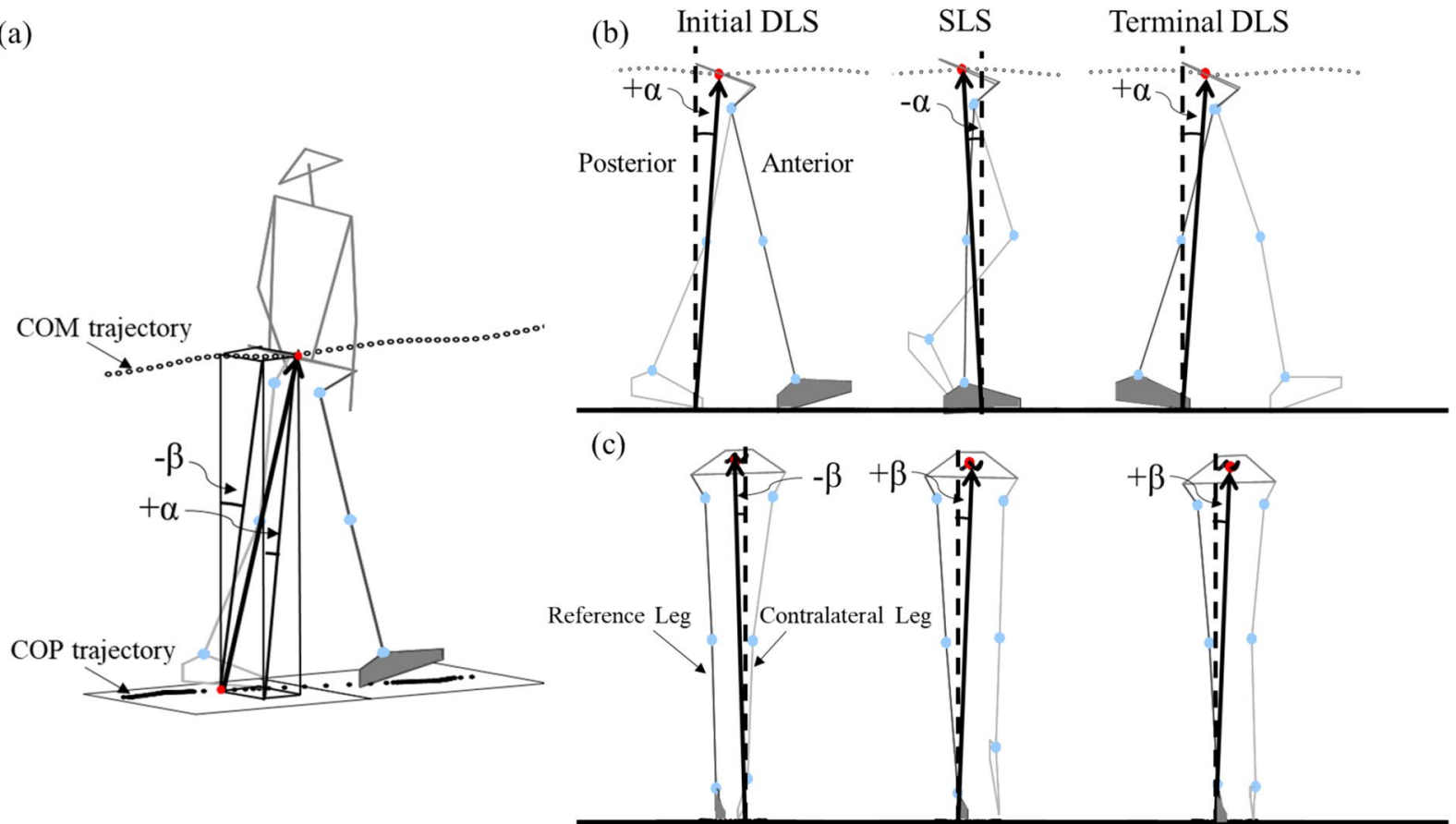

Figure 1. (a) Stick figure of the typical subject during level walking showing three-dimensional trajectories of the $\mathrm{COM}$ and $\mathrm{COP}$, and the COM-COP vector forming sagittal inclination angles $(\alpha)$ and frontal inclination angles $(\beta)$ with the vertical. The COM-COP vector and the inclination angles in the sagittal (b) and frontal (c) planes during initial and terminal double-limb support (DLS), and single-limb support (SLS) are also shown. The reference limb is shown in darker grey.

position of the whole body was calculated using the forceplate data. The IA of the COM-COP vector in the sagittal and frontal planes were then calculated as follows:

$$
\begin{gathered}
\qquad \vec{t}=\left(\vec{Z} \times \frac{\vec{P}_{C O M-C O P}}{\left|\vec{P}_{C O M-C O P}\right|}\right) \\
\text { Sagittal IA }=\sin ^{-1}\left(\mathrm{t}_{\mathrm{Y}}\right) \\
\text { Frontal IA }=\left\{\begin{array}{c}
-\sin ^{-1}\left(t_{X}\right), \text { for the right limb } \\
\sin ^{-1}\left(t_{X}\right), \text { for the left limb }
\end{array}\right.
\end{gathered}
$$

where $\vec{P}_{C O M-C O P}$ was the vector pointing from the COP to the COM, $\vec{Z}$ was the vertical unit vector, and $\vec{X}$ was the unit vector pointing in the direction of progression (Fig. 1). The RCIA were calculated by smoothing and differentiating the IA trajectories using the GCVSPL method ${ }^{30}$. A positive sagittal and frontal IA indicate that the COM is anterior to and away from the COP towards the contralateral limb, respectively. The greater the IA, the greater the horizontal projection of the COM deviates from the COP, and the greater the effort needed to maintain or reduce the deviation unless accompanied by an appropriate RCIA, corresponding to the position and velocity control of the COM as described by Pai and Patton ${ }^{31}$. All the data analyses and graphics generation in the current study were performed using programs in Matlab (R2017b, MathWorks, USA) developed in-house.

For statistical analysis, the values of the IA and RCIA in the sagittal plane and frontal plane at heel-strike and toe-off of both limbs were obtained for each trial and each subject. The ranges of IA and RCIA during the gait cycle, and time-averaged IA over the phases of initial and terminal double-limb support (DLS), single-limb support (SLS) and swing were also obtained. For each of the above-mentioned variables, data from the six trials were averaged. Each of the variables was first tested for normality using a Shapiro-Wilk test. For variables of normal distribution, the between-group differences were tested using independent $t$ tests. A significance level of $\alpha=0.05$ was set for all tests. All statistical analyses were performed using SPSS version 20 (SPSS Inc., Chicago, IL, USA).

\section{Results}

The average WOMAC subscale values for the OA group were 43 (SD 15.8) for pain, 39.2 (SD 16.9) for stiffness, and 38.3 (SD 7.5) for physical function. Compared to Control, the OA group showed significantly decreased walking speed and cadence with significantly increased step width (Table 2). No significant differences were found in the stride length and step length between the groups (Table 2). 


\begin{tabular}{|l|l|l|l|}
\hline & OA & Control & p value \\
\hline Walking speed $(\mathrm{mm} / \mathrm{s})$ & $707.4(119.1)$ & $876.8(127.13)$ & $0.001^{*}$ \\
\hline Cadence $($ steps/min) & $88.2(13.12)$ & $104.9(12.63)$ & $0.001^{*}$ \\
\hline Stride length $(\mathrm{mm})$ & $974.8(45.85)$ & $1002.7(54.61)$ & 0.141 \\
\hline Step length $(\mathrm{mm})$ & $483.9(29.45)$ & $497.4(31.12)$ & 0.231 \\
\hline Step width $(\mathrm{mm})$ & $93.9(18.39)$ & $73.7(28.9)$ & $0.030^{*}$ \\
\hline
\end{tabular}

Table 2. Means (standard deviations) of the spatiotemporal parameters during walking in the knee osteoarthritis group $(\mathrm{OA}, \mathrm{n}=15)$ and healthy controls (Control, $\mathrm{n}=15) .{ }^{\star} \mathrm{OA}$ significantly different from Control.
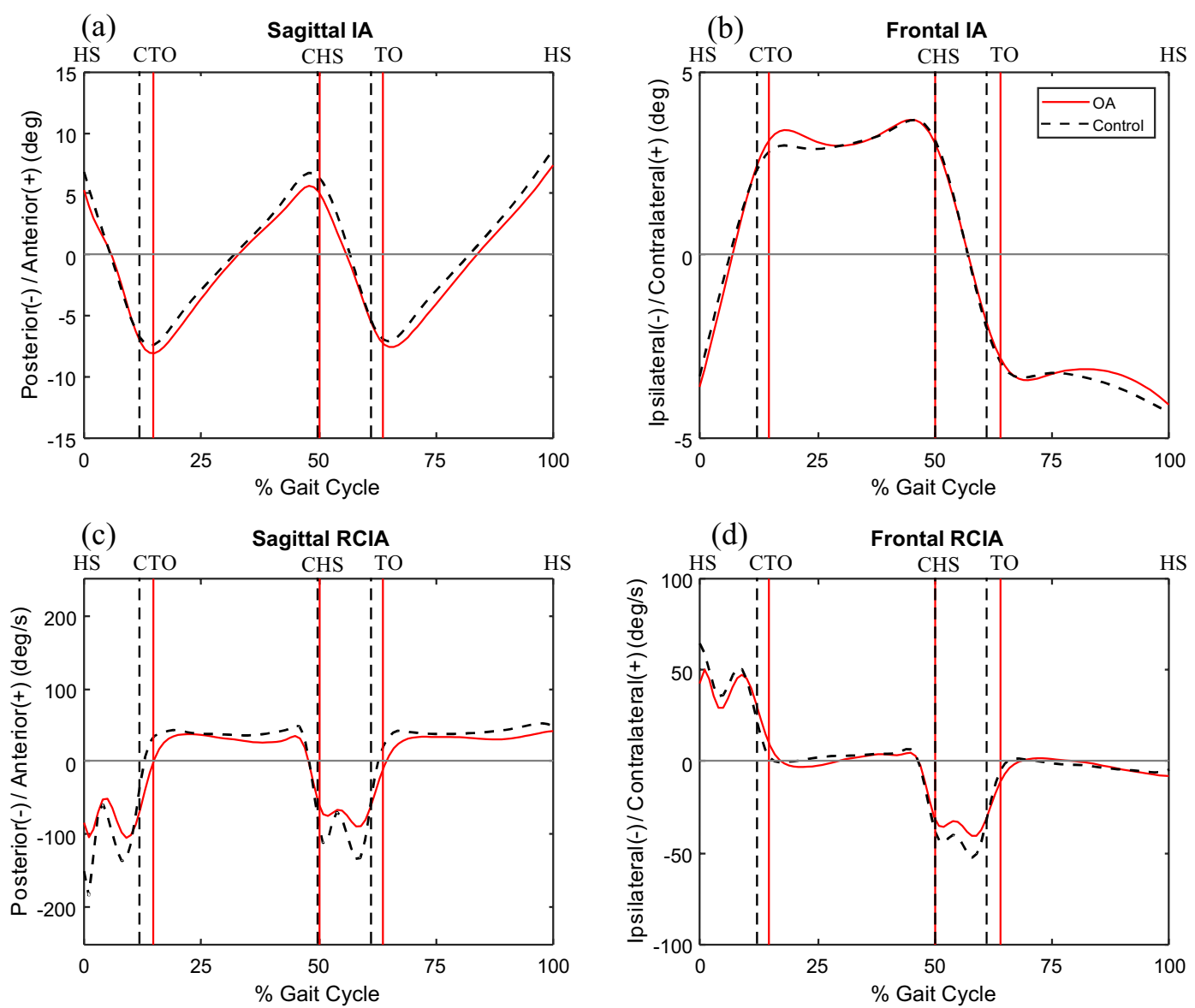

Figure 2. Mean curves of the COM-COP inclination angles (IA) and their rates of change (RCIA) in the sagittal (a,c) and frontal (b,d) planes for the knee osteoarthritis group and the control group during level walking. Gait events, namely heel-strike (HS), contralateral toe-off (CTO), contralateral heel-strike (CHS) and toe-off (TO), are indicated by vertical lines. Positive sagittal and frontal IA indicate COM positions that are anterior and contralateral to the COP, respectively. Positive sagittal and frontal RCIA indicate rates of anterior changes and contralateral changes in the corresponding IA, respectively.

Both Control and the OA group showed similar patterns in the IA curves but quite different RCIA curves between OA and Control groups, especially during DLS (Fig. 2). In the sagittal plane, compared to Control, the OA group showed significantly decreased IA at heel-strike but increased average IA during DLS (Fig. 3). The OA group also showed significantly decreased RCIA at heel-strike and toe-off, and during DLS, as well as significantly decreased average and range of RCIA during SLS and swing (Table 3; Fig. 3). In the frontal plane, compared to Control, the OA group showed significantly decreased RCIA at heel-strike and toe-off, and average RCIA during terminal DLS (Fig. 3). They also showed significantly decreased range of RCIA during SLS and swing (Table 4). 


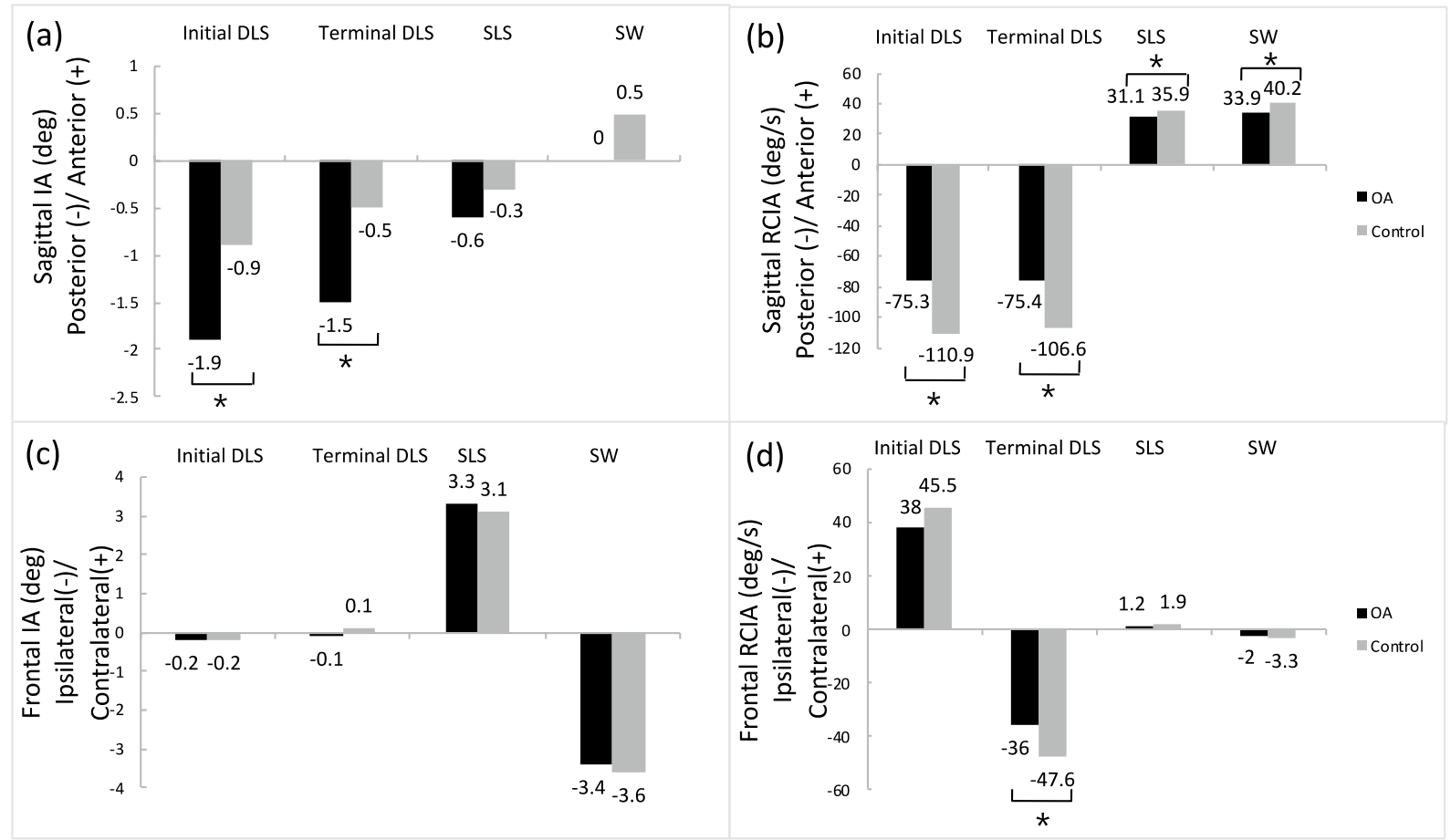

Figure 3. Mean values of time-averaged COM-COP inclination angles (IA) and their rates of change (RCIA) over phases of initial and terminal double-limb support (DLS), single-limb support (SLS) and swing during level walking in the sagittal $(\mathbf{a}, \mathbf{b})$ and frontal $(\mathbf{c}, \mathbf{d})$ planes for the knee osteoarthritis group and the control group. Positive sagittal and frontal IA indicate COM positions that are anterior and contralateral to the COP, respectively. Positive sagittal and frontal RCIA indicate rates of anterior changes and contralateral changes in the corresponding IA, respectively.

\begin{tabular}{|l|c|l|l|l|l|l|}
\hline & \multicolumn{5}{l}{ Sagittal IA (deg) } & \multicolumn{4}{l|}{ Sagittal RCIA (deg/s) } \\
\cline { 2 - 7 } & OA & Control & p value & OA & Control & p value \\
\hline HS & $6.6(0.9)$ & $7.4(1.2)$ & $0.028^{*}$ & $-41.1(41.4)$ & $-133.0(65.6)$ & $0.000^{*}$ \\
\hline CTO & $-8.3(1.1)$ & $-8.0(1.0)$ & 0.464 & $-1.9(14.3)$ & $-38.9(46.7)$ & $0.010^{*}$ \\
\hline CHS & $6.9(0.8)$ & $7.7(1.3)$ & $0.040^{*}$ & $-60.8(38.4)$ & $-119.1(53.7)$ & $0.002^{*}$ \\
\hline TO & $-8.2(0.8)$ & $-7.7(0.8)$ & 0.100 & $1.2(13.6)$ & $-40.5(29.0)$ & $0.000^{*}$ \\
\hline Range & \multicolumn{7}{|l}{} \\
\hline DLSi & $15.4(1.6)$ & $15.6(1.7)$ & 0.739 & $246.0(76.5)$ & $266.5(62.8)$ & 0.429 \\
\hline DLSt & $15.2(1.2)$ & $15.4(1.3)$ & 0.691 & $251.3(66.1)$ & $243.9(39.5)$ & 0.713 \\
\hline SLS & $15.5(1.4)$ & $16.4(1.1)$ & 0.070 & $126.4(35.7)$ & $169.6(51.3)$ & $0.000^{*}$ \\
\hline SW & $17.1(1.7)$ & $18.0(1.5)$ & 0.116 & $48.4(17.5)$ & $101.2(31.2)$ & $0.000^{*}$ \\
\hline
\end{tabular}

Table 3. Means (standard deviations) of the sagittal inclination angles (IA) and the rate of change of IA (RCIA) at heel-strike (HS), contralateral toe-off (CTO), contralateral heel-strike (CHS) and toe-off (TO), as well as average values and ranges of IAs during single-limb support (SLS), swing (SW), initial double-limb support (DLSi) and terminal double-limb support (DLSt). P-values for comparisons between knee OA and Control using independent $t$ test are also shown. All significance levels were set at $\alpha=0.05$. ${ }^{\star} \mathrm{OA}$ significantly different from Control.

\section{Discussion}

The current study aimed to identify the effects of bilateral medial compartment knee OA on the whole-body balance control during level walking, in terms of COM-COP IA and RCIA. The OA group was found to walk with an altered COM-COP control throughout the gait cycle, when compared to healthy controls. In the sagittal plane, the patients showed increased IA during DLS but decreased RCIA throughout the gait cycle, while in the frontal plane the IA was not altered but the RCIA was decreased during DLS. These results suggest that bilateral severe medial compartment knee OA compromised the COM-COP control in older adults during gait, which may be related to the increased risk of falling in this population as reported in the literature.

Patients with knee OA in the current study showed increased pain and stiffness, as well as decreased mobility during activities of daily living as indicated by the WOMAC results, in agreement with the literature ${ }^{23}$. These 


\begin{tabular}{|c|c|c|c|c|c|c|}
\hline & \multicolumn{3}{|c|}{ Frontal IA (deg) } & \multicolumn{3}{|c|}{ Frontal RCIA (deg/s) } \\
\hline & OA & Control & p value & $\mathbf{O A}$ & Control & $\mathrm{p}$ value \\
\hline HS & $-4.2(1.0)$ & $-3.5(1.1)$ & 0.092 & $26.9(19.8)$ & $50.7(20.8)$ & $0.003^{*}$ \\
\hline CTO & $3.3(0.7)$ & $2.8(0.7)$ & 0.058 & $12.0(6.4)$ & $23.7(12.6)$ & $0.003^{*}$ \\
\hline CHS & $3.9(0.9)$ & $3.7(0.9)$ & 0.452 & $-31.9(18.5)$ & $-51.4(22.4)$ & $0.015^{*}$ \\
\hline TO & $-3.4(0.8)$ & $-3.2(0.8)$ & 0.404 & $-9.7(6.8)$ & $-26.6(13.3)$ & $0.000^{*}$ \\
\hline \multicolumn{7}{|c|}{ Range } \\
\hline DLSi & $7.1(1.5)$ & $6.3(1.6)$ & 0.157 & $96.1(36.7)$ & $82.6(26.3)$ & 0.257 \\
\hline DLSt & $7.5(1.8)$ & $6.8(1.4)$ & 0.234 & $93.5(34.1)$ & $80.5(24.1)$ & 0.238 \\
\hline SLS & $1.3(0.4)$ & $1.3(0.6)$ & 0.820 & $50.0(20.4)$ & $76.5(30.0)$ & $0.009^{*}$ \\
\hline SW & $1.6(0.8)$ & $1.7(1.0)$ & 0.842 & $20.3(8.4)$ & $33.6(15.4)$ & $0.006^{*}$ \\
\hline
\end{tabular}

Table 4. Means (standard deviations) of the frontal inclination angles (IA) and the rate of change of IA (RCIA) at heel-strike (HS), contralateral toe-off (CTO), contralateral heel-strike (CHS) and toe-off (TO), as well as average values and ranges of IAs during single-limb support (SLS), swing (SW), initial double-limb support (DLSi) and terminal double-limb support (DLSt). p values for comparisons between knee OA and Control using independent $t$ test are also shown. All significance levels were set at $\alpha=0.05$. ${ }^{\star} \mathrm{OA}$ significantly different from Control.

symptoms appeared to contribute to the compromised performance of gait in these patients. For example, decreased gait speed and cadence, and increases in step width may be related to joint stiffness, limited mobility and/or attempts to minimize pain. These gait deviations also contributed directly to the changes in the control of the COM relative to the COP.

During DLS, the OA group walked with the COP moving towards the COM at reduced speed while the COM was controlled within a relatively small range, with similar or increased IA but with significantly decreased RCIA. This indicates that the OA group tended to keep the body weight longer on the trailing limb while transferring the body weight to the leading limb, helpful for reducing the loading rate and pain at the knee joint of the leading limb. It is known that an increased GRF loading rate is associated with increased pain $^{32}$. A plausible reason for this is that damaged cartilage can result in painful abrasive contact between bones ${ }^{33}$ and subsequent difficulties in both shock absorption and the structural support of the body weight. For the current patients with bilateral knee $\mathrm{OA}$, both limbs took turns to serve as the leading weight-accepting limb. Therefore, reducing RCIA during DLS appeared to be helpful for reducing the loading rate and pain at both knees during weight acceptance. However, altered body weight transfer during DLS in the sagittal plane with increased IA magnitude but without an RCIA large enough to maintain the dynamic stability of the COM (Tables 3, 4) may indicate an increased risk of loss of balance ${ }^{31}$. A brief loss of balance can lead to a devastating fall event for older people with knee OA as they may not have to ability to recover balance. On the other hand, the slower weight transfer and reduced forward propulsion during DLS seemed also to lead to reduced RCIA at the subsequent SLS, which further affected the dynamic stability of single-limb support.

During SLS, the patients with knee OA also showed a compromised COM-COP control as found during DLS, primarily in the sagittal plane with significantly decreased RCIA and range of RCIA. During SLS, the base of support is very small and the control of the COM over this small base of support is quite challenging. During this phase, the COP was controlled within a relatively small range while the body COM travelled beyond the supporting foot from a trailing position to a leading position, similar to an inverted pendulum. Reduced RCIA may indicate reduced COM velocity and angular velocity of the inverted pendulum (Tables 3, 4), which reduced the dynamic stability of the system with an increased risk of loss of balance during SLS ${ }^{31}$. In the frontal plane, the COM had a relatively small range of motion during the progression of the body over the stationary foot, with unaltered IA and RCIA. The current results suggest that the patients with knee OA were able to maintain unaltered IA and RCIA with decreased range of RCIA in the frontal plane, even though the projected COM was away from the COP most of the time. Thus, control of the stability may be more difficult to maintain than during DLS.

The current study was the first attempt to identify the effects of severe bilateral medial knee OA on the control of the body's COM motion relative to the COP during level walking. Generally, the patients with knee OA showed a compromised balance control pattern during walking, especially in the control of the rate of change of the COM to COP motion in both sagittal and frontal planes during DLS, and in the sagittal plane during SLS. While these alterations appeared to be helpful for reducing the loading rate and pain at the knee joint of the leading limb, they may also contribute to an increased difficulty in maintaining dynamic stability of COM-COP motions during walking. Therefore, monitoring for signs of increased IA, reduced RCIA and/or reduced range of RCIA during weight transfer in such patient groups is suggested for identifying an increased risk of falling, especially for those with severe medial knee OA. It is noted that the current results are limited to those with severe knee osteoarthritis. For patients with mild to moderate knee OA, further study will be needed. Another limitation is the unequal number of male and female subjects in both the OA and Control groups. While the female/male distribution of the subjects in the subject groups is a reflection of the actual situation that women have a higher prevalence of knee OA than men ${ }^{34}$, further study would be needed to test whether the current results would be affected by the sex distribution within the group. Further study on the muscle control patterns associated with the balance control strategies in the subject groups using electromyography will be useful for more insight into 
the changes of neuromuscular control following knee OA. Further studies are also needed to identify how total knee arthroplasty might affect the COM-COP control during walking.

\section{Conclusions}

The patients with severe bilateral medial knee OA showed compromised balance control with altered COM relative to COP motion when compared to healthy controls during level walking, especially reduced RCIA in the sagittal plane throughout the gait cycle, and in the frontal plane during DLS. Reducing RCIA during DLS appeared to be helpful for reducing the loading rate and pain at both knees during weight acceptance, but seemed also to lead to reduced RCIA at the subsequent SLS, further affecting the dynamic stability of SLS. Altered COM relative to COP control without an RCIA large enough to maintain the body's dynamic stability may indicate an increased risk of loss of balance. Monitoring for signs of reduced RCIA in such patient groups is suggested for identifying their increased risk of falling, especially for those with severe medial knee OA.

Received: 12 October 2020; Accepted: 1 February 2021

Published online: 12 February 2021

\section{References}

1. Felson, D. T. et al. The prevalence of knee osteoarthritis in the elderly. The Framingham Osteoarthritis Study. Arthritis Rheum. 30, 914-918 (1987).

2. Lamoth, C. J., Meijer, O. G., Daffertshofer, A., Wuisman, P. I. \& Beek, P. J. Effects of chronic low back pain on trunk coordination and back muscle activity during walking: Changes in motor control. Eur. Spine J. 15, 23-40 (2006).

3. Lamoth, C. J., Stins, J. F., Pont, M., Kerckhoff, F. \& Beek, P. J. Effects of attention on the control of locomotion in individuals with chronic low back pain. J. Neuroeng. Rehabil. 5, 13 (2008).

4. Murphy, J. W. \& Altman, R. D. Updated osteoarthritis reference standard. J. Rheumatol. Suppl. 43, 56-59 (1995).

5. Arden, N. \& Nevitt, M. C. Osteoarthritis: Epidemiology. Best Pract. Res. Clin. Rheumatol. 20, 3-25 (2006).

6. Mazzuca, S. A. et al. Effects of self-care education on the health status of inner-city patients with osteoarthritis of the knee. Arthritis Rheum. 40, 1466-1474 (1997).

7. O'Reilly, S. C., Muir, K. R. \& Doherty, M. Effectiveness of home exercise on pain and disability from osteoarthritis of the knee: A randomised controlled trial. Ann. Rheum. Dis. 58, 15-19 (1999).

8. Maurer, B. T., Stern, A. G., Kinossian, B., Cook, K. D. \& Schumacher, H. R. Jr. Osteoarthritis of the knee: Isokinetic quadriceps exercise versus an educational intervention. Arch. Phys. Med. Rehabil. 80, 1293-1299 (1999).

9. Md, J. F. B. et al. The relationship between leg power and physical performance in mobility-limited older people. J. Am. Geriatr. Soc. 50, 461-467 (2002).

10. Wang, T.-M. et al. Bilateral knee osteoarthritis does not affect inter-joint coordination in older adults with gait deviations during obstacle-crossing. J. Biomech. 42, 2349-2356. https://doi.org/10.1016/j.jbiomech.2009.06.029 (2009).

11. Levinger, P. et al. Swing limb mechanics and minimum toe clearance in people with knee osteoarthritis. Gait Posture 35, 277-281 (2012).

12. Tsonga, T. et al. Analyzing the history of falls in patients with severe knee osteoarthritis. Clin. Orthop. Surg. 7, 449-456 (2015).

13. Wegener, L., Kisner, C. \& Nichols, D. Static and dynamic balance responses in persons with bilateral knee osteoarthritis. J. Orthop. Sports Phys. Ther. 25, 13-18 (1997).

14. Duffell, L. D., Southgate, D. F., Gulati, V. \& McGregor, A. H. Balance and gait adaptations in patients with early knee osteoarthritis. Gait Posture 39, 1057-1061. https://doi.org/10.1016/j.gaitpost.2014.01.005 (2014).

15. Khalaj, N., Abu Osman, N. A., Mokhtar, A. H., Mehdikhani, M. \& Wan Abas, W. A. Balance and risk of fall in individuals with bilateral mild and moderate knee osteoarthritis. PLoS One 9, e92270. https://doi.org/10.1371/journal.pone.0092270 (2014).

16. Tinetti, M. E. \& Ginter, S. F. Identifying mobility dysfunctions in elderly patients: Standard neuromuscular examination or direct assessment?. JAMA 259, 1190-1193 (1988)

17. Lee, H.-J. \& Chou, L.-S. Detection of gait instability using the center of mass and center of pressure inclination angles. Arch. Phys. Med. Rehabil. 87, 569-575 (2006).

18. Chien, H.-L., Lu, T.-W. \& Liu, M.-W. Control of the motion of the body's center of mass in relation to the center of pressure during high-heeled gait. Gait Posture 38, 391-396. https://doi.org/10.1016/j.gaitpost.2012.12.015 (2013).

19. Paul, J. C. et al. Gait stability improvement after fusion surgery for adolescent idiopathic scoliosis is influenced by corrective measures in coronal and sagittal planes. Gait Posture 40, 510-515 (2014).

20. Huang, S.-C., Lu, T.-W., Chen, H.-L., Wang, T.-M. \& Chou, L.-S. Age and height effects on the center of mass and center of pressure inclination angles during obstacle-crossing. Med. Eng. Phys. 30, 968-975 (2008)

21. Hong, S.-W. et al. Control of body's center of mass motion relative to center of pressure during uphill walking in the elderly. Gait Posture 42, 523-528 (2015).

22. Lu, H.-L., Lu, T.-W., Lin, H.-C., Hsieh, H.-J. \& Chan, W.-P. Effects of belt speed on the body's center of mass motion relative to the center of pressure during treadmill walking. Gait Posture 51, 109-115. https://doi.org/10.1016/j.gaitpost.2016.09.030 (2017).

23. Mandeville, D., Osternig, L. R. \& Chou, L. S. The effect of total knee replacement surgery on gait stability. Gait Posture 27, $103-109$. https://doi.org/10.1016/j.gaitpost.2007.02.009 (2008).

24. Wang, T.-M., Hsu, W.-C., Chang, C.-F., Hu, C.-C. \& Lu, T.-W. Effects of knee osteoarthritis on body's center of mass motion in older adults during level walking. Biomed. Eng. Appl. Basis Commun. 22, 205-212 (2010).

25. Kellgren, J. \& Lawrence, J. Radiological assessment of osteo-arthrosis. Ann. Rheum. Dis. 16, 494 (1957).

26. Erdfelder, E., Faul, F. \& Buchner, A. GPOWER: A general power analysis program. Behav. Res. Methods Instrum. Comput. 28, 1-11 (1996).

27. Chen, H.-L. \& Lu, T.-W. Comparisons of the joint moments between leading and trailing limb in young adults when stepping over obstacles. Gait Posture 23, 69-77 (2006).

28. Chen, S.-C., Hsieh, H.-J., Lu, T.-W. \& Tseng, C.-H. A method for estimating subject-specific body segment inertial parameters in human movement analysis. Gait Posture 33, 695-700 (2011).

29. Merriaux, P., Dupuis, Y., Boutteau, R., Vasseur, P. \& Savatier, X. A study of vicon system positioning performance. Sensors 17, 1591 (2017).

30. Woltring, H. J. A Fortran package for generalized, cross-validatory spline smoothing and differentiation. Adv. Eng. Softw. 1978(8), 104-113 (1986).

31. Pai, Y.-C. \& Patton, J. Center of mass velocity-position predictions for balance control. J. Biomech. 30, 347-354 (1997).

32. Briani, R. V., Pazzinatto, M. F., Waiteman, M. C., de Oliveira Silva, D. \& de Azevedo, F. M. Association between increase in vertical ground reaction force loading rate and pain level in women with patellofemoral pain after a patellofemoral joint loading protocol. Knee 25, 398-405 (2018). 
33. Lawrence, A. et al. Synthesis and characterization of a lubricin mimic (mLub) to reduce friction and adhesion on the articular cartilage surface. Biomaterials 73, 42-50 (2015).

34. Srikanth, V. K. et al. A meta-analysis of sex differences prevalence, incidence and severity of osteoarthritis. Osteoarthr. Cartil. 13, 769-781 (2005).

\section{Acknowledgements}

The authors wish to thank Taiwan Adventist Hospital for the financial support (106-E-15).

\section{Author contributions}

P.-A.L., H.-C.L., T.-M.W. and T.-W.L. conceived and designed the experiments. P.-A.L. and K.-H.W. performed the experiments. P.-A.L., K.-H.W. and H.-Y.L. analyzed the data. P.-A.L., T.-W.L. and K.-W.S. wrote the main manuscript text. T.-W.L. and H.-C.L. contributed subjects/materials/analysis tools. All authors reviewed the manuscript. T.-W.L. and H.-C.L. are both corresponding authors.

\section{Competing interests}

The authors declare no competing interests.

\section{Additional information}

Correspondence and requests for materials should be addressed to H.-C.L. or T.-W.L.

Reprints and permissions information is available at www.nature.com/reprints.

Publisher's note Springer Nature remains neutral with regard to jurisdictional claims in published maps and institutional affiliations.

(c) (i) Open Access This article is licensed under a Creative Commons Attribution 4.0 International License, which permits use, sharing, adaptation, distribution and reproduction in any medium or format, as long as you give appropriate credit to the original author(s) and the source, provide a link to the Creative Commons licence, and indicate if changes were made. The images or other third party material in this article are included in the article's Creative Commons licence, unless indicated otherwise in a credit line to the material. If material is not included in the article's Creative Commons licence and your intended use is not permitted by statutory regulation or exceeds the permitted use, you will need to obtain permission directly from the copyright holder. To view a copy of this licence, visit http://creativecommons.org/licenses/by/4.0/.

(C) The Author(s) 2021 